\title{
Drawing and using free body diagrams: Why it may be better not to decompose forces
}

\author{
Ivica Aviani, ${ }^{1, *}$ Nataša Erceg, ${ }^{2}$ and Vanes Mešic ${ }^{3}$ \\ ${ }^{1}$ Institute of Physics, Bijenička c. 46, Hr-10002 Zagreb, Croatia and Faculty of Science, University of Split, \\ Teslina 12, 21000 Split, Croatia \\ ${ }^{2}$ Department of Physics, University of Rijeka, R. Matejčić 2, 51000 Rijeka, Croatia \\ ${ }^{3}$ Faculty of Science, University of Sarajevo, Zmaja od Bosne 33-35, 71000 Sarajevo, \\ Bosnia and Herzegovina
}

(Received 4 May 2015; published 29 December 2015)

\begin{abstract}
In this study we investigated how two different approaches to drawing free body diagrams influence the development of students' understanding of Newton's laws, including their ability to identify real forces. For this purpose we developed a 12-item two-tier multiple choice survey and conducted a quasiexperiment. This experiment included two groups of first-year physics students from Rijeka (RG) $\left(n_{e}=27\right)$ and Split (SG) $\left(n_{c}=25\right)$ Universities. Students from both groups solved mechanics problems for a period of two class hours. The only difference was that RG students used the superposition of forces approach to solving mechanics problems and in SG the decomposition of forces approach has been used. The ANCOVA ( $n_{c}=17, n_{e}=17$ ) showed a statistically significant difference in favor of RG, whereby the effect sizes were moderate to large, and the largest differences have been observed in the ability of identifying real forces. Students from the control group (SG) more often exhibited the misconception that forces and their components act on a body independently and simultaneously. Our results support the idea that the practice of resolving forces into the components may not be the most effective way to develop understanding of Newton's laws and the concept of force.
\end{abstract}

DOI: 10.1103/PhysRevSTPER.11.020137

PACS numbers: 01.40.Fk, 01.40.gb

\section{INTRODUCTION}

In earlier research it has been shown that students exhibit significant difficulties when it comes to using vectors and free body diagrams (FBDs) [1-4]. Typically, the mathematical approach to mechanics problem solving is characterized by decomposition of forces into the components. In this way additional, nonexistent forces are introduced, which leads to a loss of physical clarity. Konradtyev argues that at least for some tasks this conceptual confusion could be prevented by using the superposition of forces approach to drawing FBDs and solving mechanics problems [5]. By the superposition of forces approach we mean a direct addition of the force vectors, using the tail-to-tip method [6], without resolving the vectors into their components.

Although the quoted arguments related to the effectiveness of the superposition approach seem to be scientifically sound, as far as we know they have not yet been tested through implementation of experimental or quasiexperimental studies. So we decided to run a quasiexperimental study in order to investigate whether the superposition of forces approach results in better understanding of FBDs compared to the decomposition of forces approach.

*aviani@ifs.hr; http://aviani.ifs.hr

Published by the American Physical Society under the terms of the Creative Commons Attribution 3.0 License. Further distribution of this work must maintain attribution to the author(s) and the published article's title, journal citation, and DOI.
The main research question of this study was as follows: "Is the approach to drawing and using FBDs which avoids specifying force components more effective than the traditional approach (in which the forces are resolved into the components) when it comes to developing students' understanding of Newton's laws including their ability to identify real forces?"

By the term "real force" we mean the resultant interaction of two bodies in the considered physical situation which should be distinguished from mathematical entities such as force components.

\section{REVIEW OF RELEVANT LITERATURE}

For purposes of reducing the complexity of authentic representations and organizing the process of problem solving, physicists often use diagrams. One kind of diagram traditionally used in physics is the free body diagram (FBD). Rosengrant, van Heuvelen, and Etkina [7] define FBDs as "diagrammatic representations in which one focuses only on an object of interest and on the forces exerted on it by other objects." Most physics teachers recognize that FBDs have a high instructional value [8]. Their beliefs are supported by empirical findings that show students who correctly draw FBDs are significantly more successful in physics problem solving [7].

It is not sufficient to develop students' skills in drawing diagrams - it is also necessary to develop their abilities of using these diagrams for purposes of formulating the 
corresponding equations of motion [9]. According to Flores, Kanim, and Kautz [10] it is very important for the students to recognize that Newton's 2nd law is a relationship between vectors. To that end, students should practice the skill of finding the resultant force acting on a body and relating it to the resultant acceleration. Earlier research has shown that this task can be difficult even for physics experts [11].

Taking into account the high importance of FBDs for problem solving in mechanics, as well as the difficulties of using them in physics instruction, many researchers attempted to design effective approaches to drawing and (or) using FBDs [12-15]. All these approaches share the common characteristic of requiring the student to manipulate vectors with the final aim of facilitating problem solving (e.g., determining the acceleration of the system or some unknown force).

The procedure for solving physics problems by using FBDs usually consists of three essential steps. The first step is the construction of a free body diagram, the second step entails the graphical determination of the resultant force vector, and the third step (if required in the problem) is finding its magnitude. There are different methods for graphical vector addition [6]. The most common is the decomposition method whereby the vectors are resolved into the components and the components with the same line of action are added. Another is the superposition method where the vectors are added such that the tail of each subsequent vector is positioned at the tip of the previous vector. In both cases the magnitude of the resultant vector is determined using the Pythagorean theorem and trigonometric methods.

Some point out that the decomposition of forces approach to drawing FBDs can lead to certain difficulties in students' conceptual understanding of physics. van den Berg and van Huis [16] point out that force diagrams are often quite confusing due to a large number of arrows which are typically used to denote forces, their components, resultants, and velocities. According to Kondratyev and Sperry [5] "...activity of separation of the vector quantities into their perpendicular components adds an unnecessary complication for the student who is trying to make sense of the physical situation and solve the problem." Students often tend to "jump to force components immediately" and memorize the meanings of these components in specific cases "rather than deriving them from the geometry of the problem" [1].

Some of students' difficulties related to drawing and using FBDs probably arise due to their difficulties with manipulating vectors. Knight [2] considers vectors to be an essential component of the mathematical language of physics. At the same time he warns that, unlike physics teachers, students do not see vector manipulation as obvious. Student difficulties regarding vector manipulations (algebraic and graphical) in introductory physics courses have been also reported by
Kanim [3]. Ngyen and Meltzer [4] found that the majority of their students in the algebra-based courses were not able to correctly carry out two-dimensional vector addition, even after a full semester of study. Further, they pointed out that students do not sufficiently understand the concept of "vector direction" and are quite confused regarding the tail-to-tip and parallelogram addition rules. In the study by Barniol and Zavala [17] it has been shown that the students' choice of addition procedure depends on the context - in the context of forces students more often use the "tail-to-tail" approach, whereas in the context of displacement vectors they prefer the "tail-to-tip" approach. Faced with the relatively low student knowledge of vectors, many researchers emphasized the necessity for more practice and explicit instruction on using vectors in physics teaching [2-4].

\section{METHODS}

\section{A. Test design}

For the purposes of this study, we developed the Free Body Diagram Test (FBDT) which is provided in Appendix A (see Figs. 3 and 4). FBDT is a two-tier test that is proposed to measure students' understanding of Newton's laws (tier 1), as well as their ability to identify real forces in different mechanics contexts (tier 2). It is a multiple choice survey consisting of 12 items based on visual representation, i.e., on different FBDs. Each item stem comprises the verbal description of an object in a certain physical situation and students are required to choose one of the five graphical options that is the best match for the description. The only differences between the options are the forces acting on the body. For simplicity and also to save space we drew the forces directly on the sketch of the object [18]. Please note that such an approach is not completely in line with Rosengrant's [7] definition of FBDs to the point that in most cases the supporting surface, attached rope, or trajectory of the object are also included in the diagram. The used approach to drawing FBDs is compatible with the Croatian tradition of physics education, and in our opinion it facilitates thinking about the physical origin of forces acting on a body (by reducing cognitive load related to recalling aspects of the physical situation at hand). With the aim of excluding the influence of torques, all the forces were drawn from the object's center of mass [16]. The forces were marked by numbers. For each item, participants were required to specify which of the five diagrams correctly shows real forces acting on the body in concern (tier 1) and also to write down the names of the corresponding forces (tier 2). Thus, we could say that FBDT measures students' representational fluency [19] within the context of free body diagrams.

Our choice of item distracters was mostly based on the results of an earlier study in which we required teachers (who taught physics to seventh and eighth grade students in Croatia) to draw FBDs for situations similar to the ones 
chosen in the FBDT. Taking into account the fact that teachers exhibit similar misconceptions as their students [20] as well as the fact that many of these misconceptions could be related to the characteristics of traditional physics instruction, we could say that our test also checks for didaktikogenic misconceptions.

\section{B. Content validity of the test}

In order to provide evidence regarding the content validity of our test, we used an approach which is similar to the approach taken by Lawshe [21]. Specifically, we asked 33 high school physics teachers to answer the following questions for each of the test items:

(1a) Is the knowledge measured by this item an important part of understanding of Newton's laws of motion within the context of a typical introductory course of physics at the university level?

(1b) Is the knowledge measured by this item an important part of the ability to identify real forces in various mechanics phenomena within the context of a typical introductory course of physics at the university level?

(2) How much do you like the item on a scale from 5 (not at all) to 10 (very much)?

(3) How difficult is the item (for an average ability student) on a scale from 1 (below average difficulty) to 3 (above average difficulty)?

Based on the physics teachers' answers to the questions presented above, we obtained some quantitative measures (see Table I) related to the content validity of our test items (questions 1a and 1b), as well as about the test buy in [22] and teachers' knowledge about students (questions 2 and 3).

The results suggest that there is a very high agreement amongst physics teachers regarding the content validity of the test. The buy-in aspect of the test seems to be fully achieved. Teachers also consider the test to be at an appropriate difficulty level. For most of the items they stated that the item is at the level of a student of average ability.

The average coefficient of variation [23] for teachers' affective attitude towards the items amounted to 0.1. This result is an indicator of homogeneity regarding teachers' affections towards the items. Their estimations of an item's difficulty varied much more-the average coefficient of variation amounted to 0.3 .

\section{Participants}

The study participants were students from two different Croatian universities-University of Rijeka and University of Split. The student sample from Rijeka consisted of 27 first-year physics students. Similarly, the student sample from Split consisted of 25 first-year physics students. The gender distribution was very similar in both groups-there were 52\% males in the Rijeka group compared to 56\% males in the Split group. Both student samples have been established by means of the convenience sampling technique.

We used a two groups pre-post quasiexperimental design. Thereby, we only decided at random which of the two groups should receive the experimental treatment and which of the groups will be the control group. Specifically, Rijeka students received the experimental treatment (superposition approach to solving problems), whereby the Split students were taught based on the decomposition approach to solving problems, i.e., they were the control group.

For the purposes of this study, one of the authors of this paper gave instruction to the control group (Split), and the other author gave instruction to the experimental group (Rijeka). The Rijeka lecturer (age $=40$ ) who took part in this study has 10 years of working experience in teaching physics at the preuniversity level and 2.5 years of working experience in teaching general physics at the university level. On the other side, the Split lecturer has 8 years of experience in teaching physics at the university level, and 12 years of experience in teaching general physics at the university preparatory courses. Both teachers worked at the universities which were chosen for purposes of implementation of the research design. In order to minimize the instructor threat to the internal validity of the study, detailed lesson plans were prepared for purposes of the implementation of the experiment (see description of treatments section).

\section{Relevant characteristics of the curriculum}

The high school curriculum in Croatia explicitly proposes the teaching of superposition or decomposition of forces in the first year of secondary school (15 year olds). However, it does not include suggestions regarding the number of teaching hours which should be devoted to individual teaching units. It is important to note that in

TABLE I. Raters' opinions on FBDT-results of the teacher survey; $M$ is the mean value, SDr the standard deviation of raters' average ratings, and SDi the standard deviation of the items' average ratings; i.e., SDr is a measure of between-rater variability and SDi is a measure of between-item variability.

\begin{tabular}{lccc}
\hline \hline $\begin{array}{l}\text { Interrater agreement } \\
\text { (question 1a) }\end{array}$ & $\begin{array}{c}\text { Interrater agreement } \\
\text { (question 1b) }\end{array}$ & $\begin{array}{c}\text { Affective attitude } \\
\text { (question 2) }\end{array}$ & $\begin{array}{c}\text { Estimated difficulty } \\
\text { (question 3) }\end{array}$ \\
\hline $92.6 \%$ & $89.7 \%$ & $M=9.33$ (scale: 5-10) & $M=2.07$ (scale: $1-3)$ \\
& & SDr $=0.80$ & 0.39 \\
& & SDi $=0.10$ & SDi $=0.46$ \\
\hline \hline
\end{tabular}


Croatia the students are taught about the superposition of forces in primary school ( 7 th grade, 13 year old pupils) for the first time, whereas they do not learn about decomposition of forces until secondary school (1th grade, 15 year old pupils). Nevertheless, when faced with mechanics problems Croatian secondary school students (and teachers) prefer the decomposition of forces approach to the superposition approach [24].

At the University level, Rijeka students learn explicitly about superposition or decomposition of forces within the context of the Physics I: Mechanics course, whereby typically 3 hours of instruction are devoted to this teaching unit. Of course, students are expected to apply their knowledge about superposition or decomposition of forces in many occasions or courses throughout their study. Similarly, Split students learn about superposition or decomposition of forces within the general physics 1 course. Five hours are devoted to this topic explicitly, but students are required to apply the gained knowledge in many other courses or occasions.

\section{E. Experimental design}

As stated earlier, for purposes of answering our research question a quasiexperimental design has been implemented. Both groups were pre-tested and, after the treatment, posttested by using FBDT. In this way, we preserved the naturalistic setting, i.e., the students were in their usual teaching environment. Each student was given a copy of FBDT and a separate answering sheet (see Appendix A). Testing time was limited to $30 \mathrm{~min}$. It is important to note that students were asked to choose the diagrams that contain only the real forces and to name the forces not in accord to their function, but in accord to their physical origin.

The whole experiment, including the pretest and posttest, has been conducted as a part of regular teaching activities during the first three weeks of the second semester. In the first week students were administered the pretest. In the subsequent week they have been receiving the teaching treatment for two hours, and in the third week the students wrote the post-test.

Our independent (treatment) variable describes the treatment approach taken to handle FBDs. It consists of two levels (conditions). These conditions reflect the superposition and decomposition of forces approaches, respectively. The dependent variable is students' post-test score on FBDT and their pretest score has been used as the covariate. We attempted to make the two treatment conditions as similar as possible, except for the crucial factor, i.e., the approach to handling FBDs. Each of these two approaches is thoroughly described within the lesson plans prepared for the purposes of this study and concisely presented below. The lesson plans which were created for the two conditions differed only with respect to the taken problem solving approach. Special attention has been devoted to the issue of matching the two conditions with respect to the taught contents and examples. As emphasized earlier, the duration of the treatment was set to two teaching hours, in both groups. The students were unaware of the experimental hypothesis which contributed to minimizing the reactivity effects, thus potentially increasing the ecological validity [25].

In order to draw conclusions about the effectiveness of the teaching treatments, we used a one-way betweengroups analysis of covariance (ANCOVA). Our null hypothesis was that the post-treatment level of dependent variable, in the experimental group will be the same as in the control group. The alternative (research) hypothesis was that students who got the superposition of forces treatment will outperform students who used the decomposition of forces treatment.

\section{F. Description of treatments}

In both the experimental and control groups students were required to solve the same six physics problems, listed in Appendix B (see Fig. 5). For purposes of solving the problems, students had to draw and use FBDs. The instructors modeled the problem solving process, by explicitly demonstrating the process of drawing FBDs on the blackboard. Thereby, in the control and experimental group different approaches were used. The traditional (decomposition) approach was applied in the control group and a nontraditional (superposition) approach in the experimental group (see Appendix B).

The traditional approach to problem solving, which is typically applied in regular physics classes, most often was started as follows: The real force vector (usually gravity) was resolved into convenient components (that have the same lines of action as other forces) by using the parallelogram rule [26] and the components were added to corresponding real forces of opposite directions in order to find the resultant force (tasks 1, 2, 5, and 6). However, in some other tasks forces were directly added to corresponding forces of opposite directions in order to find the resultant force (task 3), or forces were directly cancelled with corresponding forces of opposite directions and then remaining forces were added to each other by using the parallelogram rule (task 4). The magnitude of the resultant force has been found from the geometry of the problem.

The nontraditional approach involved drawing real forces acting on the observed object, followed by their addition by applying tail-to-tip rule. As a matter of fact, we can add any two vectors by placing the tail of the second vector so that it meets the tip of the first vector. The sum is the vector from the tail of the first vector to the tip of the second vector. In our physics problems, such addition of vectors is reflected as follows: If an object is at rest or moves uniformly along a straight line, the real forces acting on the object close a polygon (see nonstandard approach in Appendix B, tasks 1 and 3). In other words, a resultant force on the observed object is zero. If an object 
accelerates, the real forces acting on it do not close the polygon, and the resultant force extends from the tail of the first vector to the tip of the last vector, closing the polygon of forces (see nonstandard approach in Appendix B, tasks $2,4,5$, and 6 ), in accordance with the tail-to-tip rule. In this case the magnitude of the resultant force is calculated from the side lengths of the obtained polygon (mostly triangle) of forces.

\section{RESULTS}

\section{A. Item statistics and test reliability}

Detailed FBDT item statistics for pretest and post-test data from the student survey together with the average item difficulty as estimated by the raters (i.e., teachers) is given in Table II. Specifically, Table II contains item difficulty indices (proportions of correct answers) and item discrimination indices for the first tier and both tiers, separately. Mean values and standard deviations are given in the last column.

In the relevant literature, item discrimination index values below 0.2 are considered to be low [27]. Based on this criterion, we found that only item 1 is potentially problematic for the first tier, as well as for both tiers. This item should be revised in future studies. There is also a problem with the second tier of item 11 due to a proper naming of the centripetal force which will be discussed later.

The reliability of the test has been estimated by calculating the Kuder-Richardson 20 (KR-20) measure [28]. From the pretest data we found values of 0.73 for the first tier and 0.77 for both tiers. From the post-test data we found values of 0.67 for the first tier, and 0.78 for both tiers. Typically, KR-20 values above 0.7 are considered to be satisfactory [29]. Taking into account the low number of test items in FBDT, we find the obtained values to be satisfying, i.e., the obtained data suggests that FBDT's internal consistency is relatively good.

A statistically significant, strong negative correlation between raters estimations of item difficulty and empirically observed item difficulty index has been found (estimations vs pretest: $r=-0.699, p=0.011$; estimations vs post-test: $r=-0.63, p=0.028$ ). This means that the high school teachers estimated item difficulties very well.

\section{B. Comparison of overall gain}

It is important to note that 10 out of 27 cases (students) in the Rijeka group, and 8 out of 25 cases in the Split group, did not write both the pretest and the post-test. Consequently, all of these cases were excluded from the ANCOVA. Generally,

TABLE II. The FBDT item statistics: item difficulty and item discrimination indices, based on the results of the pretest and post-test survey. The raters' estimates of item difficulties are also given for purposes of comparison.

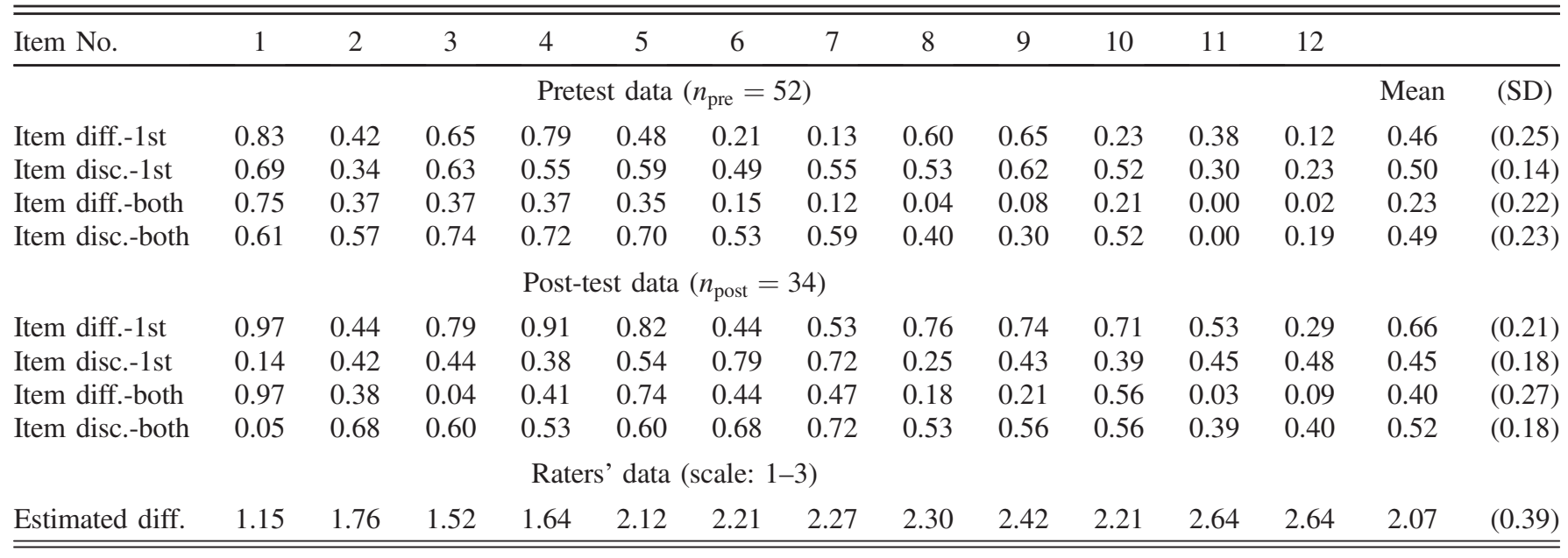

TABLE III. Pretest and post-test average scores and information about gain. Standard errors are given in parenthesis.

\begin{tabular}{|c|c|c|c|c|}
\hline & \multicolumn{2}{|c|}{ 1st tier } & \multicolumn{2}{|c|}{ Both tiers } \\
\hline & $\begin{array}{c}\text { Rijeka group } \\
\text { (experimental) }\end{array}$ & $\begin{array}{l}\text { Split group } \\
\text { (control) }\end{array}$ & $\begin{array}{c}\text { Rijeka group } \\
\text { (experimental) }\end{array}$ & $\begin{array}{c}\text { Split group } \\
\text { (control) }\end{array}$ \\
\hline & $n_{e}=17$ & $n_{c}=17$ & $n_{e}=17$ & $n_{c}=17$ \\
\hline Pretest score & $4.18(0.46)$ & $6.94(0.53)$ & $2.00(0.49)$ & $3.59(0.60)$ \\
\hline Post-test score & $7.76(0.63)$ & $8.12(0.56)$ & $5.29(0.75)$ & $4.41(0.59)$ \\
\hline Pre-post gain & $3.59(0.4)$ & $1.18(0.44)$ & $3.29(0.59)$ & $0.82(0.41)$ \\
\hline Between group gain difference & \multicolumn{2}{|c|}{$2.41(0.6), p<0.001, r=0.58$} & \multicolumn{2}{|c|}{$2.47(0.72), p<0.01, r=0.52$} \\
\hline Hake's normalized gain & 0.46 & 0.23 & 0.33 & 0.1 \\
\hline
\end{tabular}




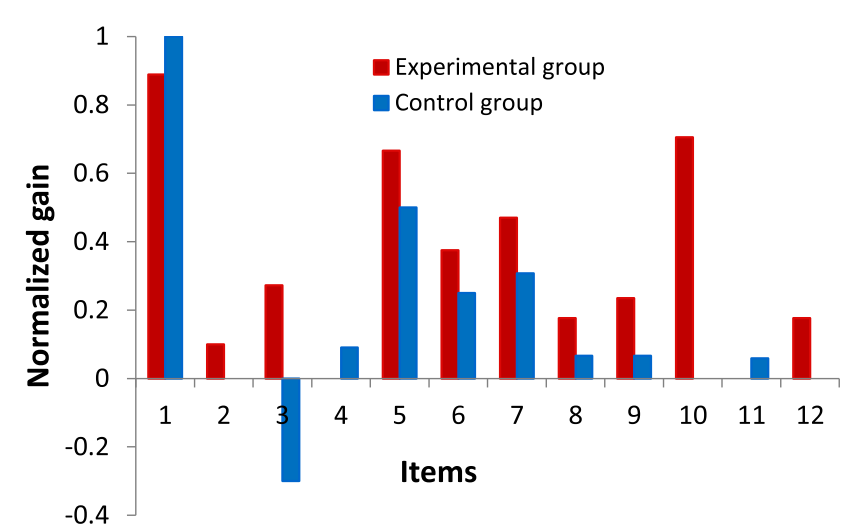

FIG. 1. Between group comparison of Hake's normalized gains for individual items (both tiers).

the existence of baseline differences represented a threat to the internal validity of our study. In order to alleviate that threat we enriched our data analysis by using the normalized gain measure. Pretest and post-test average scores and information about gain are given in Table III.

The calculation of the between group gain difference is based on formulas given by McNemar [30]. The effect size $r$ is calculated based on the $t$-test value in line with the instructions given by Rosnow, Rosenthal, and Rubin [31].

In order to explore the relative efficacy of the interventions for specific physical situations it is useful to compare the gains for individual test items. The normalized gain measures [32] (both tiers) are shown in Fig. 1.

\section{The ANCOVA}

ANCOVA was conducted to compare the effectiveness of two different interventions. The independent variable was the type of intervention. The dependent variable consisted of students' scores on the FBDT after the interventions were completed and their pre-intervention scores on the same test were used as the covariate. Students' answers to an item were coded with one only if they were correct on both tiers of the item, i.e., only if they chose the right option and were able to correctly identify all the forces acting on the body or bodies of interest.

We checked the assumptions of normality, homogeneity of variances, and homogeneity of regression slopes, the assumption of homogeneity of variance, and the assumption of normally distributed errors. All these assumptions were met for our data. Further, the KR-20 for pretest and post-test scores proved to be satisfactory, which means that the reliability of measurements for the dependent variable as well as for the covariate was relatively high.

The covariate, pretest scores, was significantly related to the students' post-test scores, $F(1,31)=25.81, p<0.001$, $r=0.67$. There was also a significant effect of intervention on post-test scores after controlling for the effect of pretest scores, $F(1,31)=8.33, p<0.01$, and $r=0$, 46. In other words, the experimental treatment resulted with significantly higher post-test scores compared to the control treatment.

\section{Analysis of student answers on individual items}

For purposes of identifying students' preconceptions related to our dependent variable, we analyzed the answers from all the 52 students who wrote the pretest. Table IV contains the most frequently chosen incorrect student answers, as well as the corresponding percentages and specifics of the chosen diagrams. These data were particularly useful for identifying student misconceptions related to the 1st and 2nd Newton's law, as well as for gaining some insight about students' conceptual confusion regarding the relationship between components and resultant forces (see discussion section).

Some useful information about student thinking about forces could also be derived from analyzing their answers on tier 2, i.e., from their naming of forces (see Table V). While the information from tier 1 allowed us to draw some conclusions about student understanding of Newton's 1st

TABLE IV. The most frequently chosen incorrect answers in the pretest $(N=52)$, the corresponding percentages and specifics.

\begin{tabular}{lccl}
\hline \hline Task & Most frequent incorrect answer & Overall percentage & Specifics of the chosen diagram \\
\hline 1 & A & $15 \%$ & Normal force is missing. \\
2 & B & $17 \%$ & Normal force is missing. \\
3 & A and C & Normal force is missing. Resultant force is not zero \\
& & & vector as expected in uniform linear motion. \\
4 & D & $8 \%$ & Normal force is missing. \\
5 & E & $25 \%$ & Components of gravity are unnecessary involved. \\
6 & B & $48 \%$ & Components of gravity are unnecessary involved. \\
7 & A & $62 \%$ & Components of gravity are unnecessary involved. \\
8 & B & $25 \%$ & Normal and gravitational force are missing. \\
9 & C & $21 \%$ & Normal and gravitational force are missing. \\
10 & D & $42 \%$ & Components of gravity are unnecessary involved. \\
11 & E & $27 \%$ & Centrifugal force is unnecessary involved. \\
12 & E & $54 \%$ & Resultant force is not centripetal force, \\
& & & but force in the direction of motion. \\
\hline \hline
\end{tabular}


and 2nd law, the information from tier 2 brought some insight about students' understanding of between-object interactions and their understanding regarding actionreaction force pairs, which allowed us to draw some conclusions about students' understanding of Newton's 3rd law. In addition, we got a more detailed picture of students' ideas regarding the relationship between forces, components, and resultants.

In order to present an as-wide-as-possible spectrum of force namings, we took into account pretest as well as post-test data. The pretest-post-test comparison of incorrect namings for several types of forces is given in Fig. 2.

\section{DISCUSSION}

\section{A. Discussion about the relative efficacy of the experimental and control treatment}

The results of the gain comparison for the control and experimental group (see Table III) as well as the results of the ANCOVA indicate that we should reject the null

TABLE V. List of correct names of forces, tasks in which the corresponding forces appear in the correct diagrams, and examples of the corresponding incorrect namings of forces on pretest and post-test.

\begin{tabular}{|c|c|c|c|c|}
\hline \multirow[b]{2}{*}{$\begin{array}{l}\text { Correct force } \\
\text { name }\end{array}$} & \multicolumn{4}{|c|}{ Examples of incorrect force namings in the correctly chosen diagrams } \\
\hline & $\begin{array}{l}\text { Tasks in } \\
\text { which } \\
\text { the force } \\
\text { appears }\end{array}$ & $\begin{array}{l}\text { Namings which } \\
\text { indicate } \\
\text { difficulty in } \\
\text { understanding } \\
\text { between-object } \\
\text { interactions }\end{array}$ & $\begin{array}{l}\text { Namings which } \\
\text { indicate } \\
\text { difficulty in } \\
\text { distinguishing } \\
\text { between forces, } \\
\text { components, and } \\
\text { resultants }\end{array}$ & Other namings \\
\hline $\begin{array}{l}\text { Gravitational } \\
\text { force }\left(\vec{F}_{g}\right)\end{array}$ & $1-12$ & $\begin{array}{l}\text { Force on the } \\
\text { surface from the } \\
\text { body }\end{array}$ & & $\begin{array}{l}\text { Gravitational potential force; } \\
\text { relative force }\end{array}$ \\
\hline $\begin{array}{l}\text { Normal } \\
\text { force }\left(\vec{F}_{N}\right)\end{array}$ & $1-9,11$ & $\begin{array}{l}\text { Reaction force of } \\
\text { gravity }\end{array}$ & & $\begin{array}{l}\text { Body resistance; tension in the cord; } \\
\text { gravitational potential force; force } \\
\text { that causes a motion; force in the } \\
\text { opposite direction of gravity; } \\
\text { gravitational force; frictional force; } \\
\text { centripetal force; centrifugal force }\end{array}$ \\
\hline \multirow[t]{2}{*}{$\begin{array}{l}\text { Frictional } \\
\text { force }\left(\vec{F}_{f}\right)\end{array}$} & $2-7$ & $\begin{array}{l}\text { Reaction force of } \\
\text { the body }\end{array}$ & $\begin{array}{l}\text { Component of } \\
\text { gravity }\end{array}$ & $\begin{array}{l}\text { Pulling force; force in the opposite } \\
\text { direction of motion; force } 2 \text {; normal } \\
\text { force; force of rest; force of } \\
\text { motion; force of slope }\end{array}$ \\
\hline & $8-9$ & $\begin{array}{l}\text { Pulling force } \\
\text { from the left } \\
\text { man; reaction } \\
\text { force of the } \\
\text { body }\end{array}$ & & $\begin{array}{l}\text { Weight; resistance force to rope; } \\
\text { tension in the cord; force F; force } \\
\text { of interaction; normal force }\end{array}$ \\
\hline $\begin{array}{l}\text { Pulling } \\
\text { force }\left(\vec{F}_{p}\right)\end{array}$ & $3-4$ & & $\begin{array}{l}\text { Resultant; force } \\
\text { that accelerates } \\
\text { the body }\end{array}$ & $\begin{array}{l}\text { Gravitational force; force in the } \\
\text { direction of acceleration or motion; } \\
\text { inertia; friction; force of motion; } \\
\text { surface; force } F \text {; velocity; } \\
\text { resistance; force that causes a } \\
\text { change in motion; force direction }\end{array}$ \\
\hline $\begin{array}{l}\text { Tension } \\
\text { force }\left(\vec{F}_{T}\right)\end{array}$ & $8-9$ & $\begin{array}{l}\text { Pulling force of } \\
\text { the body }\end{array}$ & & $\begin{array}{l}\text { External force; force in the direction } \\
\text { of motion; force of motion; reaction } \\
\text { force; potential force; force of } \\
\text { interaction; friction; normal force; }\end{array}$ \\
\hline & 10 & & & $\begin{array}{l}\text { Pulling force; resistance; surface } \\
\text { resistance }\end{array}$ \\
\hline $\begin{array}{l}\text { Normal force of } \\
\text { the groove }{ }^{\mathrm{a}}\left(\vec{F}_{g}\right)\end{array}$ & $11-12$ & & Centripetal force ${ }^{\mathrm{b}}$ & $\begin{array}{l}\text { Gravitational force; fractional force; } \\
\text { pulling force; radial force }\end{array}$ \\
\hline
\end{tabular}

${ }^{\mathrm{a}}$ The contact force between the walls of the channel and the ball. Actually it is normal force but occurs tangentially to the plane of motion because of the shape of the groove.

${ }^{b}$ Many students failed to realize that centripetal force is the resultant force that is directed toward the center of the curvature of the path-they often think that individual forces acting toward the center of the curvature of the path are centripetal forces on their own, which is true only in certain circumstances. 


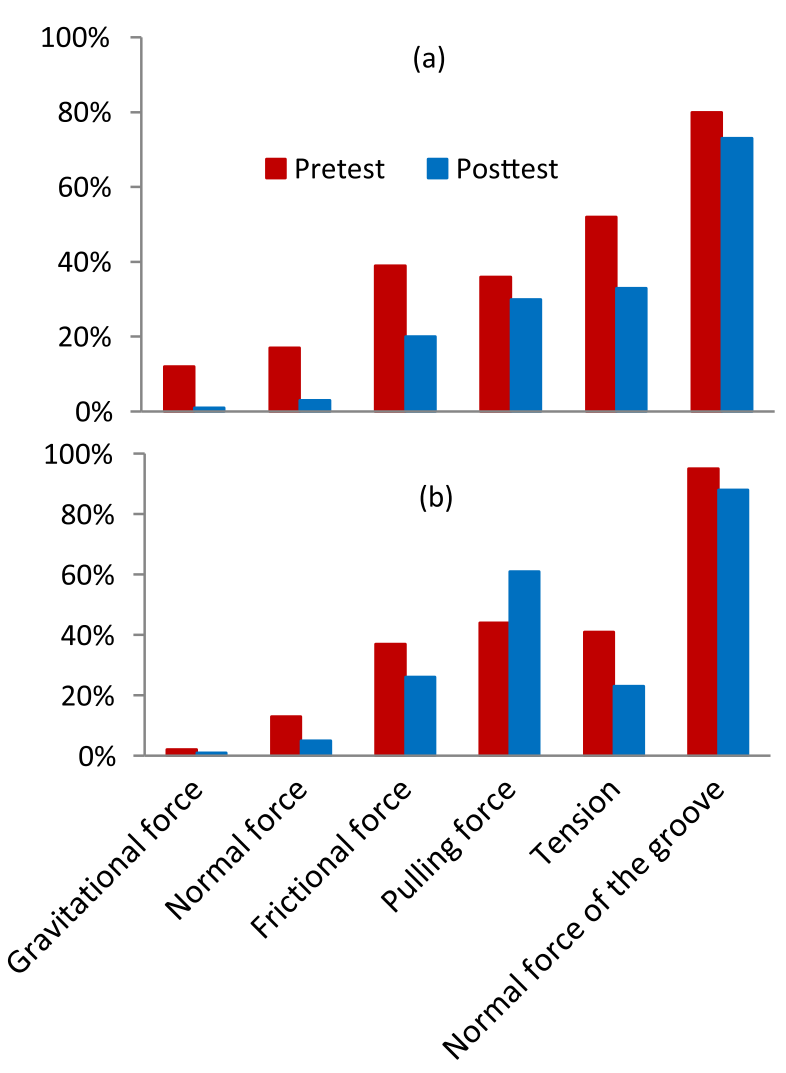

FIG. 2. Percentage of occasions in which the first tiers of the corresponding items have been correctly solved, but the force has been named incorrectly or not named at all: (a) experimental group, (b) control group.

hypothesis and accept the alternative hypothesis according to which students who get the superposition of forces treatment outperform the students who get the decomposition of forces treatment when it comes to developing students' understanding of Newton's laws including their ability to specify real forces.

These results seem to support the ideas by Kondratyev and Sperry [5], who advocated for the direct use of vectors in mechanics problems and criticized the practice of resolving forces into the components prior to applying Newton's laws. Specifically, while analyzing the disadvantages of the decomposition of vectors approach to solving mechanics problems, they pointed out that in extreme cases the student may think that nature does not exist without coordinate axes. By overemphasizing the decomposition of forces procedure, we run the risk that many of our students do not differentiate between real forces and some mathematical, auxiliary entities (e.g., force components). Exactly this has been shown in our study, where students from the experimental group outperformed their peers from the control group with respect to the ability of identifying real forces acting on physical objects. Savinainen et al. [33] found that "the likelihood of the correct identification of forces in an FBD is relatively high if the student has identified interactions correctly in an interaction diagram."
In our opinion, our approach as well as the approach by Savinainen et al. [33] strives at focusing attention on physical aspects of a given situation, instead of putting accent on mathematical procedures.

For 9 out of 12 test items, the normalized gains were larger in the experimental group compared to the control group (see Fig. 1). Largest normalized gain differences in favor of the experimental group have been observed for items 10 and 3. In item 10 (see Appendix A), students had to identify forces acting on a pendulum, which are only the gravitation force and the tension in the rope. None of the students from the experimental group solved this item correctly in the pretest context. They mostly chose the distractor D (65\%) which is an indicator for the misconception according to which a gravitational force as well as its components act on the pendulum, at the same time. After the treatment, the percentage of correct answers in the experimental group increased from $0 \%$ to $71 \%$ which suggests that our experimental treatment helped the students to overcome the described misconception.

In the control group there was no pretest-post-test gain on item 10. The distractor which has been most frequently chosen in the control group was distractor D (29\%) on the pretest and on the post-test distractor C (29\%), where tension of the rope, gravity force, and their resultant are shown. Students who chose that distractor seem to believe that there are three forces acting on the pendulum bob-the two real forces and their resultant. In other words, it seems as if the decomposition approach to solving problems may be not the most effective way for overcoming the misconception related to "thinking that individual forces and their resultant act on a body at the same time." One factor which could have additionally motivated the students to pick distractor $\mathrm{C}$ is related to the widely known misconception that there always has to be one force acting in the direction of motion of the object.

In item 3 (see Appendix A) students had to choose the FBD which best describes an object that moves with constant velocity across a horizontal surface. The results of the pretest showed that in the experimental group students mostly chose distractors A and C. In the control group, students mostly chose distractors A, C, and D. All of these distractors imply that there is nonzero net force acting on the body. These results clearly show that students do not adequately associate the force diagram with the application of Newton's 1st law. The choice of the distractor C is most probably related to the famous "motion requires force" misconception [34]. After the treatment, the percentage of students who exhibited the described misconception in the experimental group decreased from $18 \%$ to $0 \%$.

Typically, in the superposition approach students develop the habit to explicitly apply Newton's laws in their vectorial form, i.e., they are expected to consistently approach the procedure of finding the resultant force vector by adding the force vectors which makes it possible to 
reason about the net acceleration vector. In the case of distractor A the resultant force vector would be directed towards the supporting surface which is counterintuitive. This could explain the fact that students from the experimental group had a much larger gain on item 3, compared to their peers from the control group. In fact, the percentage of students who chose answer A decreased from $18 \%$ to $6 \%$ in the experimental group. The reason for a negative gain in the control group could be related to the fact that on the post-test fewer students were able to correctly specify forces acting on the body in comparison to the pretest, i.e., to the inefficacy of the treatment with respect to the ability on tier 2 rather than on tier 1 . This also indicates that in the decomposition of forces approach students more often fail to differentiate between the real forces and some mathematical abstractions.

For items 1, 4, and 11 the normalized gain was larger for students from the control group. However, the effect size corresponding to these differences was very small. The largest (although small) normalized gain difference, in favor of students from the control group, was observed for item 1 . In this item students had to identify the diagram which best describes a body which rests on a horizontal surface. Even at pretest $94 \%$ of control group students solved it correctly compared to $47 \%$ students from the experimental group. At post-test all of the students from the control group solved this item correctly, compared to $94 \%$ students from the experimental group. Therefore, we conclude that the normalized gain was very large in the control group, mostly because it reached the theoretical maximum. Generally, due to its low discrimination value (see Table II) which is related to its easiness, item 1 will be probably discarded from future versions of the test.

Finally, it should be noted that the results of normalized gain analyses reinforced our ANCOVA findings, i.e., they also showed that the experimental treatment seems to be superior in comparison to the control treatment when it comes to our dependent variable.

\section{B. Discussion about incorrect answers and corresponding misconceptions}

In order to gain further insight into student preconceptions, as well as into the diversity of students' ideas related to Newton's laws and the concept of force, we decided to take a closer look at student responses to the FBDT items.

In items 5, 6, 7, and 10, students often chose incorrect force diagrams that unnecessarily involved components of the gravitational force. This result supports our assumption that the use of the decomposition of forces solving method creates a misconception that the force components are real forces too, acting simultaneously with the original force. As a matter of fact, by analyzing the student responses we found that many students lack the ability to distinguish among the real forces, the components of the forces, and the resultant force. For example, as seen from Table V, there are some students who have chosen the correct force diagrams, but they incorrectly named a frictional force as a component of gravitational force, or a pulling force and the normal force of the groove as a resultant or centripetal force. Note that the resultant force is not a name for a real force. The students' failure to distinguish between forces, components, and resultant is probably caused by confusing approaches to drawing FBD on blackboards, as well as in textbooks [16]. Such confusing diagrams contain arrows for forces, arrows for components of forces, and an arrow for a resultant or net force, at the same time. Even when teachers and textbook authors use different colors for components and the resultant, students usually do not use colors when drawing diagrams. Consequently, when trying to learn from their lecture notes, students often are not able to distinguish between forces, components, and resultants. Thus, difficult physics concepts are made even more difficult. To facilitate discernment, in our research treatments we drew forces as simple arrows, components of forces as dashed arrows, and the resultant force as a double-line arrow.

The most frequent incorrect answers in items 3 and 12 indicate the existence of the well-known misconception that continuing motion implies the presence of a continuing net force in the direction of the motion. Concretely, in item 3 , one of the most common incorrect answers was the answer $\mathrm{C}$, in which the resultant force is not zero as it must be for uniform linear motion. It has been found that many students exhibit this misconception even after they have completed their course in mechanics, and that it shows up in a wider diversity of problem situations than one would expect [34]. Similarly, the most common incorrect answer in item 12 is related to choosing a diagram in which the resultant force is not directed to the center of the circle, as it should be for a uniform circular motion, but in the direction of motion.

In item 11, the most frequent incorrect answer was the answer E, where the centrifugal force was unnecessarily added. Specifically, it seems as if students often treat circular motion as a kind of equilibrium, whereby the equilibrium is established between a centripetal force and an equal but opposite centrifugal force [35]. The "counterbalancing" notion of circular motion has already been noted by Viennot [36].

Most common incorrect answers in items 1, 2, 3, and 4 clearly show that students often chose incomplete force diagrams, in which the normal force is missing. Obviously, students are fully aware of the force of gravity, which always acts on the body in concern, but they are not aware of the presence of the normal force which must exist to keep the body at rest. This reflects students' difficulties in understanding of Newton's 1st law. The reason for this could also lie in textbooks, where we often find incomplete force diagrams containing only the forces which are of particular interest for a certain problem. For example, for a body on an inclined plane authors often draw only the 
gravitational force and its components acting on the body. In our opinion such an approach may contribute to the development of the misconception that gravitational force and its components act on a body independently at the same time. By analyzing students' incorrect namings of forces in the correctly chosen force diagrams for each physical situation (Table V), we also came up with some conclusions regarding student understanding about between-object interactions, as well as about their ability to identify action-reaction force pairs, which can be related to student understanding of Newton's 3rd law. For example, some students have mixed up the gravitational force exerted on the body by the Earth with the contact force by which the body acts upon the supporting surface due to gravity. As a matter of fact, for a body on a horizontal surface students often chose the correct diagram, which showed two forces acting on the same body along the vertical axis. However, some students designated the two forces as a "force on the surface from the body" and "normal force" of the surface. We know that these two contact forces represent an actionreaction pair for the described situation. Consequently, it seems that these results indicate that some of our students exhibit the misconception according to which forces of action and reaction act on the same body. Similarly, the frictional force exerted on a man (in items 8 and 9) by the supporting surface is considered to be his pulling force.

Regarding the mentioned names of forces, the graph in Fig 2 shows the improvement of students' post-test results in comparison to their pretest results, except for the pulling force. However, the percentage of incorrect namings is still relatively high, especially for those forces that are rarely mentioned in class. Trying to eliminate these difficulties, Turner [37] and Savainen et al. [33] suggest applying system schemas or interaction diagrams in physics classes. These visual representations are considered helpful in situations where student misconceptions frequently lead to specifying incorrect numbers of forces, misidentification of the agents of the forces, or an incorrect application of Newton's 3rd law.

\section{SUMMARY AND CONCLUSION}

Vector calculus, in most physics courses, is carried out by the traditional algebraic methods, i.e., by resolving the forces into the components and then summing up the components that have the same line of action. This method has some advantages, particularly in the treatment of a large number of vectors and complicated geometry. After resolving the forces into the components only a simple algebraic operation of adding numbers is needed for summing up the components with the same line of action and obtaining the resultant. In this way a geometrical problem is transformed to an algebraic problem, which facilitates solving even for those students who are less talented in geometry. However, in this procedure the number of the vectors appearing in the force diagram is typically very high.
Although decomposing forces facilitates calculation, the introduction of additional vectors in the diagram makes it difficult for the students to grasp the physical aspects which are at the very core of the corresponding problem. Students often develop a misconception that components are also real forces that can act on objects simultaneously with real forces (resultant interactions between two bodies). In addition, the procedure of decomposing force vectors can result in students' conceptual confusion when it comes to application of Newton's laws that commonly state that the motion of the body is determined by the vector sum of the forces acting on the body. The question arises: Is the approach to drawing and using FBDs, which avoids specifying force components, more effective than the traditional approach (in which the forces are resolved into the components) when it comes to developing student understanding of Newton's laws, including their ability to identify real forces?

For purposes of answering this question we developed a multiple choice two-tier questionnaire consisting of 12 items that is intended to measure student understanding of Newton's laws, including the ability to correctly specify real forces. This questionnaire proved to be a valid and reliable instrument.

In order to compare the efficacy of the two problem solving approaches we conducted a quasiexperimental study. Our test was administered to the two groups of first-year physics students at University of Rijeka (experimental group, $n_{e}=27$ ) and University of Split (control group, $n_{c}=25$ ), after the concept of force had been covered in class. The treatments consisted of solving the same problems in both groups for a period of two class hours, whereby a similar level of guidance has been used in both groups. The only difference between the control and experimental treatment was that in the experimental group the teacher's scaffolding of drawing FBDs and students' problem solving did not include decomposing, but only superposition of forces. We measured student understanding before and after the treatments.

The normalized average gain in the experimental group was 0.46 , compared with 0.23 in the control group. The results of the ANCOVA showed a statistically significant difference in favor of the experimental group, whereby the effect size proved to be moderate to large. This result is obviously in line with the argument of Kondratyev and Sperry [5] who suggest direct use of vectors in mechanics problems instead of resolving forces into the components.

It is important to note that the results of our study indicate that the use of the decomposition of forces method to drawing and using FBDs may result with the misconception that a force and its components can act on a body at the same time. Besides that, our results additionally reinforce the findings from earlier studies regarding the existence of the robust preconception that motion of a body implies the existence of force acting on that body along the direction of its motion [34]. 
For some FBDs, students named forces which in reality represent action-reaction pairs for the corresponding physical situations, showing thereby difficulties in understanding Newton's 3rd law.

We believe that the superposition of forces approach has the following advantages: (a) using only one rule for summing vectors, (b) drawing only real forces on FBD, (c) clearly presenting $\sum_{i} \vec{F}_{i}=\overrightarrow{0}$ or $\sum_{i} \vec{F}_{i}=m \vec{a}$ for direct application of Newton's laws, (d) developing a geometric way of thinking, and (e) preventing the development of certain misconceptions.

The main limitation of this study is related to the fact that the teacher who gave the treatment was not the same for the control and experimental group. However, in our opinion this circumstance was not a serious threat to the internal validity of our study, because we created very detailed lesson plans for the two treatment groups, in order to make the lessons as little as possible dependent on a (individual) teachers' spontaneous actions. The fact that the instructor of the control group had more university teaching experience and very rich references in popularization of physics further decreases the threat to internal validity.

An additional limitation of the study is related to the relatively small number of students in the treatment groups. However, taking into account the fact that our data did not violate the assumptions of ANCOVA (homogeneity of variance, homogeneity of regression slopes, normality assumption), we can consider the results of our statistical testing as reliable. As a matter of fact, the number of students per group is bigger than 15 , which is considered to be a minimum sample size required for experimental research [38].

In our future work, we are going to run some think-aloud studies in order to further improve our understandings of the huge differences between groups on certain FBDT items. This would allow us to get additional insight into the cognitive effects of the described approaches and to plan further theoretical and practical improvements of the problem solving approach described in this paper. Besides implementing think-aloud studies, it would be also be interesting to investigate how the effectiveness of the described teaching treatments depends on the participants' level of representational fluency.

\section{ACKNOWLEDGMENTS}

The authors would like to thank all the participants who took part in this study. Special thanks to Mladen Buljubašić for organizing the primary school physics teachers survey and to Lucija Krce for her help in implementing the FBDT. I. A. was supported by the Ministry of Science of Republic of Croatia, under the bilateral Croatia-USA agreement on the scientific and technological cooperation, Project No. 1/2014. N. E. acknowledges the financial support of the University of Rijeka for scientific research under the Project No. 13.12.1.4.06 "Physical properties of circumstellar matter in symbiotic stars."

\section{APPENDIX A: FREE BODY DIAGRAM TEST}

See Figs. 3 and 4.

Dear participant, by carefully answering this questionnaire you participate in a scientific research which is aimed at improvement of physics teaching. Before you proceed, please fill the identification form which contains information that is important for our study:

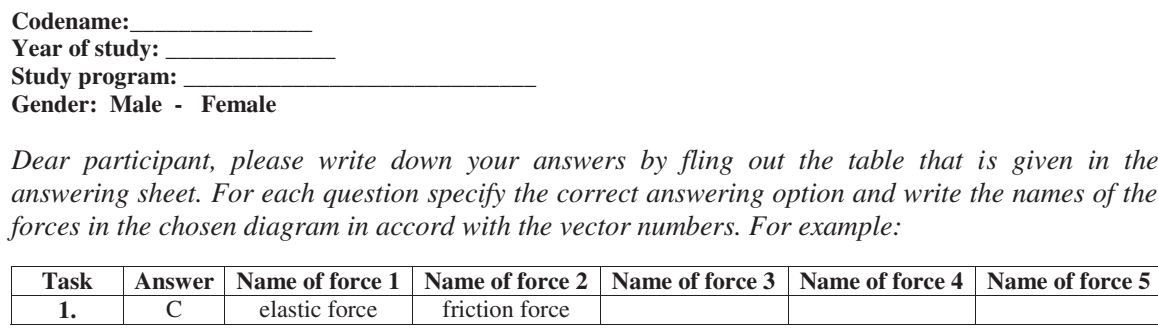

FIG. 3. Written instructions given to the students. Additional instructions and explanations were given to the students orally. They were asked to choose the diagrams that contain only the real forces and to name the forces not in accord to their function, but in accord to their physical origin. 


\section{Questionnaire}

1. A block is resting on a horizontal surface. Which of the following diagrams correctly shows the forces acting on the block?

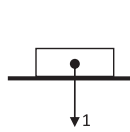

(a)

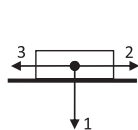

(b)

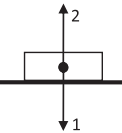

(c)

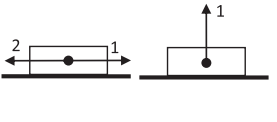

(d)
2. The pulling force $F$ is acting on a block, but the block is still at rest Which of the following diagrams correctly shows the forces acting on the block?

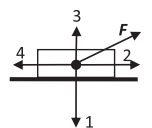

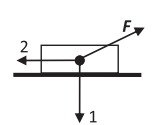

(b)

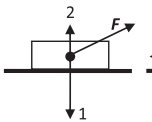

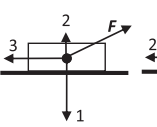

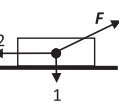

3. A block is sliding along a horizontal surface at constant speed. Which of the following diagrams correctly shows the forces acting on the block?

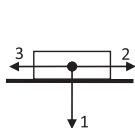

(a)

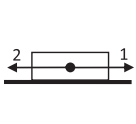

(b)

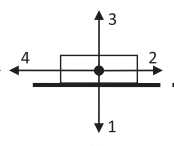

(c)

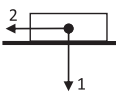

(d)

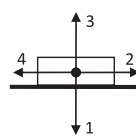

(e)
4. A block is accelerating along a horizontal surface. Which of the following diagrams correctly shows the forces acting on the block?

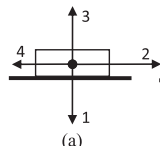

(a)

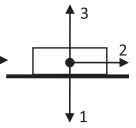

(b)

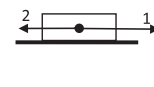

(c)

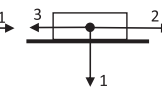

(d)

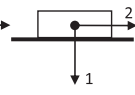

(e)
5. A block is resting on an inclined plane. Which of the following diagrams correctly shows the forces acting on the block?

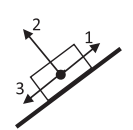

(a)

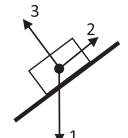

(b)

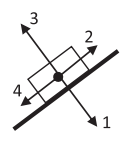

(c)

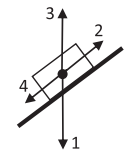

(d)

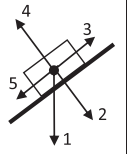

(e)
6. A block is sliding down an inclined plane at constant speed. Which of the following diagrams correctly shows the forces acting on the block?
7. A block is sliding down an inclined plane at constant acceleration.

Which of the following diagrams correctly shows the forces acting on block?

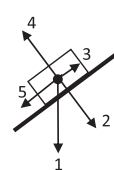

(a)

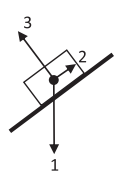

(b)

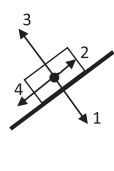

(c)

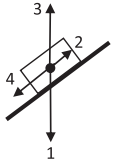

(d)

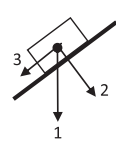

(e)
8. Two men are resting while they are pulling the rope between them. Which of the following diagrams correctly shows the forces acting on the left man?

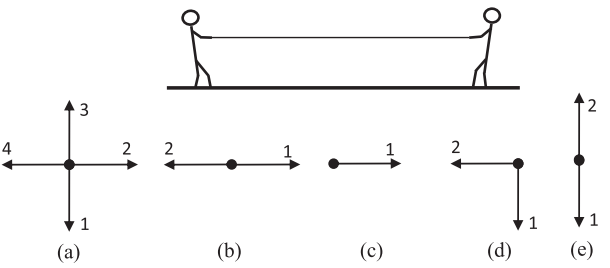

9. Two men are pulling the rope between them. In doing so, the stronger man (on the right side of the rope) is at rest, and the weaker man (on the left side of the rope) is sliding to the right. Which of the following diagrams correctly shows the forces acting on the left man when his motion starts?

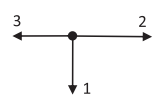

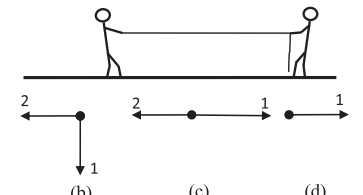

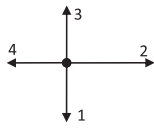

(e)

10. The pendulum bob is swinging from right to left, starting from a position higher than the one shown in figure. If we neglect air resistance, which of the following diagrams correctly shows the forces acting on the pendulum bob?

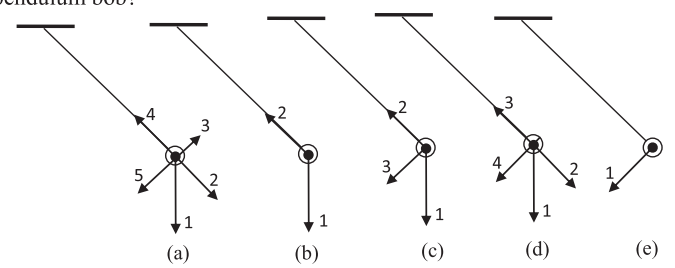

11. The ball is in circular clockwise motion along a horizontal channel. If we neglect friction and air resistance, which of the following diagrams correctly shows the forces acting on the ball?

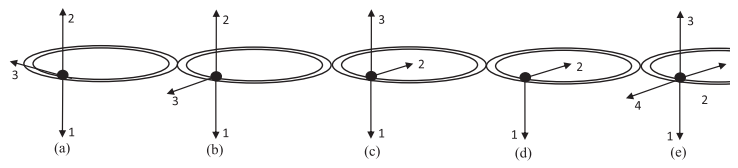

12. The ball is in a clockwise motion along a vertical circular loop. If we neglect friction and air resistance, which of the following diagrams correctly shows the forces acting on the ball?

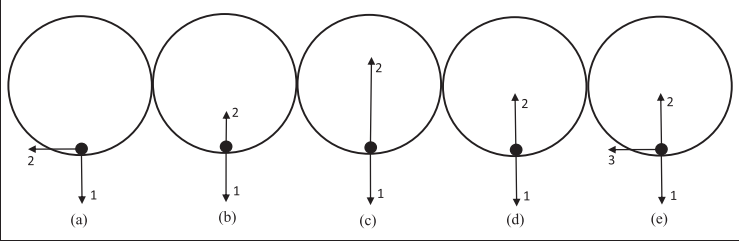

FIG. 4. Free body diagram questionnaire. 


\section{APPENDIX B: PHYSICS PROBLEMS SOLVED IN CLASS}

See Fig. 5.

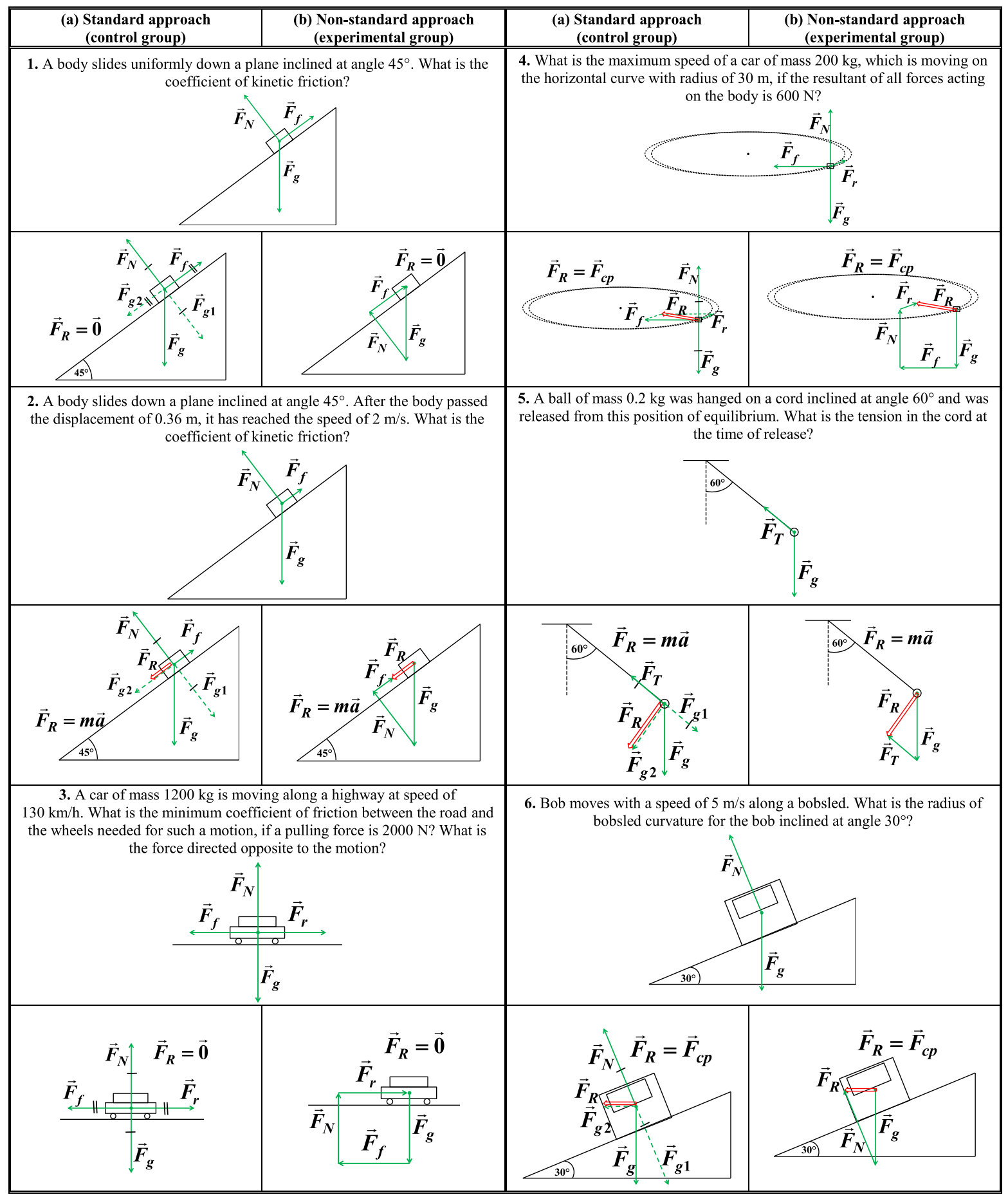

FIG. 5. Physics problems solved in class. For each of the six physics problems given in the Table, the standard (a) and nonstandard (b) approach to drawing FBDs is shown. Forces are drawn as simple arrows, components of forces as dashed arrows, and the resultant force as a double-line arrow. Pairs of vectors that cancel out are marked by single or double bars on the arrows. 
[1] S. Flores-García, L. L. Alfaro-Avena, J. E. Chávez-Pierce, J. Luna-González, and M. D. González-Quezada, Students' difficulties with tension in massless strings, Am. J. Phys. 78, 1412 (2010).

[2] R. D. Knight, The vector knowledge of beginning physics students, Phys. Teach. 33, 74 (1995).

[3] S. E. Kanim, Ph.D. dissertation, University of Washington (1999).

[4] N.-L. Nguyen and D. E. Meltzer, Initial understanding of vector concepts among students in introductory physics courses, Am. J. Phys. 71, 630 (2003).

[5] A. S. Kondratyev and W. Sperry, Direct use of vectors in mechanics problems, Phys. Teach. 32, 416 (1994).

[6] D. C. Giancoli, Physics for Scientists and Engineers with Modern Physics, 4th ed. (Pearson Education Inc., SAD, 2009).

[7] D. Rosengrant, A. van Heuvelen, and E. Etkina, Do students use and understand free-body diagrams? Phys. Rev. ST Phys. Educ. Res. 5, 010108 (2009).

[8] P. Wendel, Adding value to force diagrams: Representing relative force magnitudes, Phys. Teach. 49, 308 (2011).

[9] K. Fisher, Exercises in drawing and utilizing free-body diagrams, Phys. Teach. 37, 434 (1999).

[10] S. Flores, S. E. Kanim, and C. H. Kautz, Student use of vectors in introductory mechanics, Am. J. Phys. 72, 460 (2004).

[11] F. Reif and S. Allen, Cognition for interpreting scientific concepts: A study of acceleration, Cognit. Instr. 9, 1 (1992).

[12] M. Mattson, Getting students to provide direction when drawing free-body diagrams, Phys. Teach. 42, 398 (2004).

[13] D. P. Maloney, Forces as interactions, Phys. Teach. 28, 386 (1990).

[14] A. Puri, The art of free-body diagrams, Phys. Educ. 31, 155 (1996).

[15] W. Sperry, Placing the forces on free-body diagrams, Phys. Teach. 32, 353 (1994).

[16] E. van den Berg and C. van Huis, Drawing forces, Phys. Teach. 36, 222 (1998).

[17] P. Barniol and G. Zavala, Vector addition: Effect of the context and position of the vectors, in Proceedings of the 2010 Physics Education Research Conference, Vol. 1289 (Portland, Oregon, 2010), pp. 73-76.

[18] J. E. Court, Free-body diagrams revisited-i, Phys. Teach. 37, 427 (1999).

[19] M. Hill, M. D. Sharma, J. O’Byrne, and J. Airey, Developing and evaluating a survey for representational fluency in science, Int. J. Innovation Sci. Math. Educ. 22, 22 (2014).

[20] J. J. Mintzes, J. H. Wandersee, and J. D. Novak, Research on alternative conceptions in science, in Handbook of
Research on Science Teaching and Learning, edited by D. L. Gabel (MacMillan, New York, 1994), pp. 177-210.

[21] C. H. Lawshe, A quantitative approach to content validity, Personnel psychology 28, 563 (1975).

[22] S. B. McKagan, K. K. Perkins, and C. E. Wieman, Design and validation of the quantum mechanics conceptual survey, Phys. Rev. ST Phys. Educ. Res. 6, 020121 (2010).

[23] D.C. Howell, Statistical Methods for Psychology (Wadsworth, Belmont, CA, 2013).

[24] I. Aviani, Kako možemo pokazati da se sile zbrajaju kao vektori, Matematičko-fizički list LXIII, 189 (2013).

[25] R. B. Johnson and L. B. Christensen, Educational Research: Quantitative, Qualitative, and Mixed Approaches (SAGE Publications, Inc., Thousand Oaks, CA, 2012).

[26] To add vectors $\boldsymbol{a}$ and $\boldsymbol{b}$ using the parallelogram method, place the tail of $\boldsymbol{b}$ so that it meets the tail of $\boldsymbol{a}$. Take these two vectors to be the first two adjacent sides of a parallelogram, and draw in the remaining two sides. The vector sum, $\boldsymbol{a}+\boldsymbol{b}$, extends from the tails of $\boldsymbol{a}$ and $\boldsymbol{b}$ across the diagonal to the opposite corner of the parallelogram.

[27] P. Kline, A Handbook of Test Construction: Introduction to Psychometric Design (Methuen, New York, NY, 1986).

[28] G. F. Kuder and M. W. Richardson, The theory of the estimation of test reliability, Psychometrika 2, 151 (1937).

[29] R. L. Doran, Basic Measurement and Evaluation of Science Instruction (National Science Teachers Association, Washington, DC, 1980).

[30] Q. McNemar, Psychological Statistics (John Wiley and Sons, Inc., New York, 1966).

[31] R. L. Rosnow, R. Rosenthal, and D. B. Rubin, Contrasts and correlations in effect-size estimation, Psychol. Sci. 11, 446 (2000).

[32] R. R. Hake, Interactive-engagement versus traditional methods: A six- thousand-student survey of mechanics test data for introductory physics courses, Am. J. Phys. 66, 64 (1998).

[33] A. Savinainen, A. Mkynen, P. Nieminen, and J. Viiri, Does using a visual-representation tool foster students ability to identify forces and construct free-body diagrams? Phys. Rev. ST Phys. Educ. Res. 9, 010104 (2013).

[34] J. Clement, Students preconceptions in introductory mechanics, Am. J. Phys. 50, 66 (1982).

[35] P. Gardner, Circular motion: Some post-instructional alternative frameworks, Res. Sci. Educ. 14, 136 (1984).

[36] L. Viennot, Spontaneous reasoning in elementary dynamics, Eur. J. Sci. Educ. 1, 205 (1979).

[37] L. Turner, System schemas, Phys. Teach. 41, 404 (2003).

[38] M.D. Gall, J.P. Gall, and W. R. Borg, Educational Research: An Introduction 7th ed. (Pearson Education, Boston, 2003). 